https://doi.org/10.15407/ujpe64.10.938

P. NOWIK-BOLTYK, ${ }^{1}$ I.V. BORISENKO ${ }^{1,2}$ V.E. DEMIDOV, ${ }^{1}$ S.O. DEMOKRITOV ${ }^{1}$

${ }^{1}$ Institute for Applied Physics and Center for Nanotechnology, University of Münster (Corrensstrasse 2-4, 48149 Münster, Germany; e-mail: demokrit@uni-Münster.de)

2 Kotel'nikov Institute of Radio Engineering and Electronics, Russian Academy of Sciences (125009 Moscow, Russia)

\title{
MAGNON LASER
}

\begin{abstract}
We experimentally demonstrate a magnon laser based on the coherent Bose-Einstein condensate of magnons brought into motion by using a time-dependent spatially inhomogeneous magnetic field. We show that the application of a short field pulse results in the formation of a condensate cloud moving with the constant velocity of $930 \mathrm{~m} / \mathrm{s}$ for the used parameters of the experiment. The number of magnons building the cloud is not changed during the propagation, which is reminiscent of the magnon superfluidity.
\end{abstract}

Keywords: magnon laser, Bose-Einstein condensate of magnons, yttrium iron garnet.

Bose-Einstein condensate of magnons (mBEC) discovered in 2006 [1] in Yttrium Iron Garnet (YIG) films at room temperature describes a state of the magnon gas characterized by the macroscopic number of magnons occupying the minimum-frequency spectral state. Since the group velocity of magnons at the minimum frequency is zero, the mBEC does not move in space, although the phase velocity of the condensed magnons can be as large, as several kilometers per second. In this paper, we present an approach, which allows one to put a coherent condensate into the constant-velocity motion. In this way, we implement a magnetic analog of an optical [2] or atom laser [3-5]. Although there are different more or less restrictive definitions of atom lasers, we follow that proposed by Robins et al. [6]: "a device that produces a guided or freely propagating beam of bosons that have been out-coupled from one or more macroscopically-occupied trapped modes". In fact, atomic and magnon condensates possess a lot of similarities: in both cases, a quasiequilibrium bosonic gas is formed either by the cooling and thermalization of atoms [3] or by injection of additional magnons and their thermalization [7, 8]. An atom laser utilizes the transfer of trapped atoms into an untrapped state by $\mathrm{RF}$ radiation $[9,10]$. In a magnon system, the condensate can be brought into motion by shifting it away from the state with zero group veloc-

(C) P. NOWIK-BOLTYK, I.V. BORISENKO, V.E. DEMIDOV, S.O. DEMOKRITOV, 2019 ity, by using a time-dependent locally inhomogeneous magnetic field. It is important to note that atom and magnon lasers differ dramatically from optical lasers, as the dispersion is concerned. The dispersion of optical laser pulses is very weak, since, for light, the dependence of the frequency on the wavevector is almost linear. On the contrary, the dispersion of atom and magnon laser pulses is significant, since the corresponding dependence is parabolic.

Figure 1 shows the schematics of the experiment. As the magnetic medium, we use a $6 \mu \mathrm{m}$-thick film of YIG epitaxially grown on a Gadolinium Gallium Garnet (GGG) substrate. The mBEC is created by the injection and the following thermalization of subthermal magnons and is detected by using the Brillouin light scattering (BLS) spectroscopy $[1,7,11]$. A strong microwave pumping field necessary for the magnon injection is created by a dielectric resonator with the resonant frequency $f_{\text {res }}=9.055 \mathrm{GHz}$ and the quality factor of about 100. A pumping power of about 1 Watt was continuously applied to the resonator. The device is placed into a uniform static magnetic field $B_{0}=66 \mathrm{mT}$. Due to the spatial non-uniformity of the microwave field of the resonator, the condensate is confined in a region in the middle of the resonator with a lateral size of about $200 \mu \mathrm{m}$, where the pumping field is strong enough to inject a critical number of magnons necessary for the formation of mBEC.

To create an additional time-dependent spatially inhomogeneous magnetic field, we use a gold conductor (width $d=10 \mu \mathrm{m}$, thickness $h=400 \mathrm{~nm}$, and

ISSN 2071-0194. Ukr. J. Phys. 2019. Vol. 64, No. 10 
length $l=8 \mathrm{~mm}$ ) fabricated on a sapphire plate, which is placed between the resonator and the YIG sample (Fig. 1). A short pulse of the electrical current in the conductor creates a local Oersted field. The $z$ component of the Oersted field locally increases or decreases the total magnetic field depending on the direction of the current flow.

In the first step, we examine the effects of the inhomogeneous field on the condensate above the conductor, as illustrated by Fig. 2 showing the BLS intensity proportional to the density of the condensate. First, the field is lowered by $\Delta B=6.25 \mathrm{mT}$ by applying a current pulse at $t \approx 0$ with a rise/fall time $\tau=500 \mathrm{~ns}$ and the duration at maximum of $T=500$ ns (white dashed line in Fig. 2, a). As seen in Fig. 2, $a$, the condensate experiences the frequency shift $\Delta f=160 \pm 30 \mathrm{MHz}$. This value is in good agreement with that expected from the variation of the total field: $\Delta f_{t h}=\gamma \Delta B=175 \mathrm{MHz}$, where $\gamma$ is the gyromagnetic ratio of an electron. After the end of the current pulse, the frequency of the condensate returns to its initial value. A similar process was studied by Rezende et al. [12] for travelling backward volume spin waves. As illustrated in Fig. 2, $b$, the behaviors change drastically, when the local field is increased. Although the frequency of the condensate first follows the variation of the field, its density strongly decreases with time. At the moment, when the field reaches its maximum value, the condensate completely disappears. Only after the end of the field pulse, the condensate can be observed again. Additional measurements described below show that, in reality, the condensate does not disappear. Instead, it is pushed away from the conductor by the hill-like inhomogeneous magnetic field.

We study the spatio-temporal dynamics of the condensate, by using time- and space-resolved BLS. We map the condensate density as a function of the time and the distance from the center of the conductor. To emphasize the spatio-temporal dynamics of the condensate, we use a smaller duration of the field pulse and the rise/fall times $T=30 \mathrm{~ns}$ and $\tau=10$ ns. Figure 3, a shows snapshots of the spaceresolved one-dimensional normalized profiles of the condensate density recorded at different delay times with respect to the start of the field pulse, as labelled. These data clearly show that the condensate cloud is pushed away from the area of the inhomogeneous magnetic field. The cloud keeps moving even
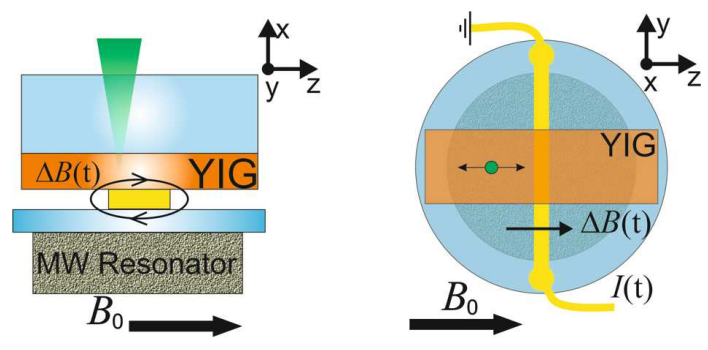

Fig. 1. Schematics of the experiment. Pumping field is produced by a dielectric resonator excited by a microwave source (not shown). The pumping injects magnons into the YIG film placed on the top of the resonator. After the thermalization, the magnons form mBEC. A gold conductor on a sapphire plate is placed between the resonator and the YIG film in order to create a time-dependent inhomogeneous magnetic field $\Delta B$ locally modifying the uniform magnetic field $B_{0}=66 \mathrm{mT}$. The condensate is detected using BLS. The spatial dependence of the condensate density is recorded by scanning the probing laser spot along the $z$ direction

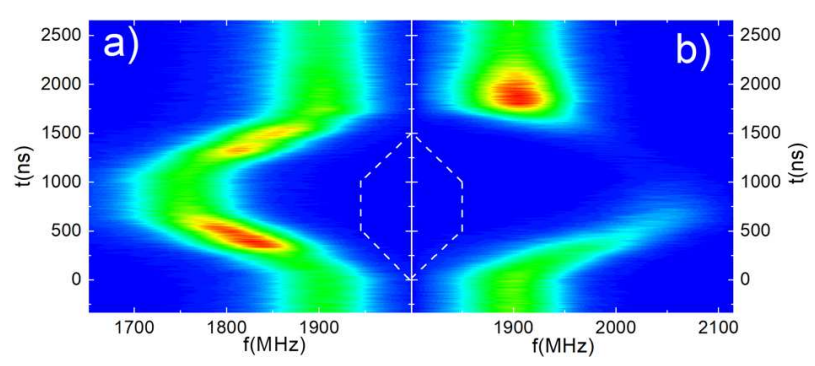

Fig. 2. Color-coded dependences of the condensate density in the time-frequency coordinates recorded above the conductor. Case of the locally decreased field. Case of the locally increased field $(a)$. The data were obtained at $\Delta B=6.25 \mathrm{mT}$. The temporal traces of the field pulse are shown by white dashed curves $(b)$

after the end of the field pulse. During the motion, the spatial width of the cloud increases due to the magnon dispersion. Figure $3, b$ shows the temporal dependences of the position of the center of the cloud and of its spatial halfwidth at the half maximum determined from the Gaussian fit of the measured profiles. The data clearly show that the cloud propagates with a constant velocity, as emphasized by the straight dashed line. From the slope of the line, we obtain the condensate velocity of $930 \mathrm{~m} / \mathrm{s}$. Additional experiments show that this velocity monotonically increases with $\Delta B$. It is important to note that the integral magnon density shows almost no variation during the propagation, as illustrated in Fig. 3,c). In other words, the total number of magnons in the 


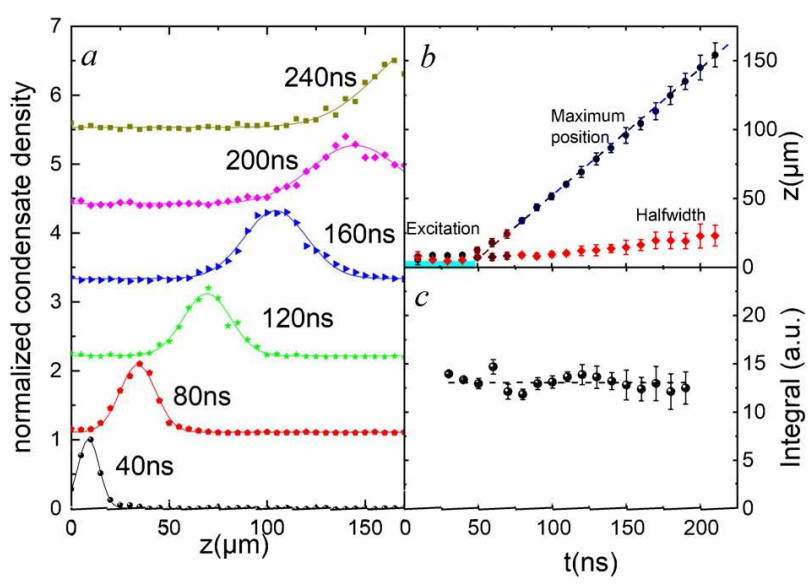

Fig. 3. Spatial profiles of the condensate density measured at $B_{0}=66 \mathrm{mT}$ and $\Delta B=6.25 \mathrm{mT}$ at different delays with respect to the start of the field pulse, as labelled $(a)$. Position of the center of the magnon cloud and its spatial width as a function of the propagation time. The straight line halfwidth shows the linear fit of the experimental data, emphasizing the constant-velocity movement of the cloud $(b)$. Temporal dependence of the total number of magnons in the cloud obtained from the integration of the condensate density. Dashed horizontal line is a guide for the eye $(c)$

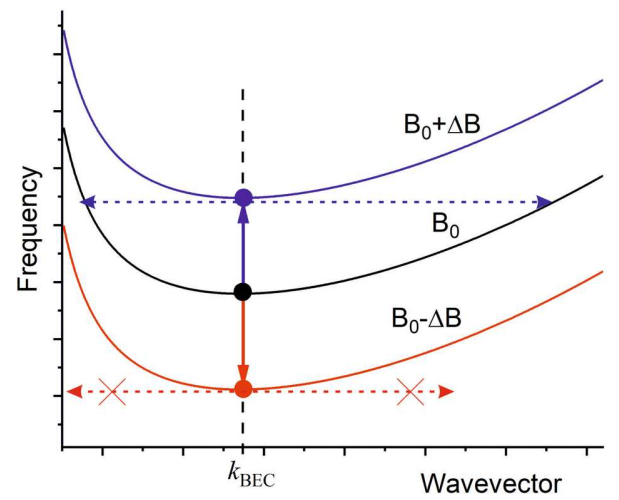

Fig. 4. Calculated magnon dispersion curves for three different values of the magnetic field. Middle curve: dispersion at the field $B_{0}$ without additional inhomogeneous field. Upper curve: dispersion at the field $B=B_{0}+\Delta B$. Lower curve: dispersion at $B=B_{0}-\Delta B$. The circles mark the lowest-frequency spectral states corresponding to the equilibrium condensate at the corresponding field values. Arrows illustrate the transition of the condensate into states with non-zero group velocity resulting in its motion

cloud remains constant within the experimental accuracy. The observed behaviors (constant velocity and constant number of magnons in the cloud) might be interpreted as an indication of magnon superfluid- ity. However, one should take into account that the moving condensate cloud is not isolated. In fact, it receives additional magnons due to the continuous pumping by the microwave field and the following condensation of the pumped magnons, which compensates the magnon decay due to the damping. Nevertheless, it is obvious from the experimental data that the moving cloud remains in a certain quasiequilibrium state described by a constant number of magnons.

To gain a further insight into the physical processes underlying the creation of a moving condensate cloud and determining the operation of a magnon laser, we consider the magnon dispersion spectrum in a vicinity of the minimum-frequency state (Fig. 4). The middle curve in Fig. 4 shows the dispersion curve for magnons at $B_{0}$, which describes magnons in the absence of an inhomogeneous field $\Delta B$ created by the current in the conductor. It is also applicable at all times for magnons outside of the conductor area. The corresponding circle indicates the spectral state with the wavevector $k_{\mathrm{BEC}}$, at which the condensate is formed before the current pulse is applied. The lower curve is the dispersion curve at $B_{0}-\Delta B$. It describes magnons close to the center of the conductor, when the field is lowered, whereas the corresponding circle marks the spectral state of the condensate at this field. Note that this state corresponds to the global minimum of the condensate frequency in all spatial positions. Therefore, a local decrease of the field creates a potential well trapping the condensate. Moreover, due to the gradient of the potential, one expects a flow of the condensate toward the center of the conductor resulting in an increase of the condensate density directly above the conductor, in agreement with the experimental data shown in Fig. 2, a). The upper curve in Fig. 4 is the dispersion curve at $B_{0}+\Delta B$. It characterizes magnons close to the center of the conductor, when the field is increased, whereas the corresponding circle marks the spectral state of the condensate at this field. Apparently, the situation in the case differs dramatically from that in the case of the local field reduction. The frequency of the condensate above the conductor is higher than that in the surrounding film. As a result, the condensate can evolve into states with the wavevectors which are larger and/or smaller than $k_{\mathrm{BEC}}$ possessing a non-zero group velocity (lower arrows in Fig. 4). It is important to note that this 
evolution does not require changes in the energy of magnons building the condensate. In other words, a local increase of the magnetic field results in an outcoupling of magnons from the condensate and creation of a moving cloud of condensed magnons, i.e., the realization of a magnon laser.

In conclusion, we have experimentally demonstrated an approach, which allows one to bring a magnon Bose-Einstein condensate into the motion, by using a local variation of the magnetic field. We show that the generated condensate cloud moves with a constant velocity, and the number of magnons in the cloud remains constant during the propagation. The observed phenomenon has a striking analogy with an atom laser. This finding demonstrates the fact that ensembles of bosons can behave in a similar way irrespectively of whether they are built by matter particles (atoms [3, 9, 10], molecules [13]), elementary particles (photons $[2,14]$ ), or quasiparticles in solids (excitons [15, 16], polaritons [17], and magnons [18, 19]).

This work was supported in part by the Deutsche Forschungsgemeinschaft (project number 416727653). S.O.D and V.E.D acknowledge the numerous stimulating discussions with G.A. Melkov on the nature of magnon $B E C$.

1. S.O. Demokritov, V.E. Demidov, O. Dzyapko, G.A. Melkov, A.A. Serga, B. Hillebrands, A.N. Slavin. Bose-Einstein condensation of quasi-equilibrium magnons at room temperature under pumping. Nature 443, 430 (2006).

2. T.H. Maiman. Stimulation optical radiation in a ruby. $\mathrm{Na}$ ture 187, 493 (1960).

3. M.H. Anderson, J.R. Ensher, M.R. Matthews, C.E. Wieman, E.A. Cornell. Observation of Bose-Einstein condensation in a dilute atomic vapor. Science 269, 198 (1995).

4. H.M. Wiseman. Defining the (atom) laser. Phys. Rev. A 56, 2068 (1997).

5. M. Holland, K. Burnett, C. Gardiner, J.I. Cirac, P. Zoller. Theory of an atom laser. Phys. Rev. A 54, R1757 (1996).

6. N.P. Robins, P.A. Altin, J.E. Debs, J.D. Close. Atom lasers: Production, properties and prospects for precision inertial measurement. Phys. Rep. 529, 265 (2013).

7. V.E. Demidov, O. Dzyapko, S.O. Demokritov, G.A. Melkov, A.N. Slavin. Thermalization of a parametrically driven magnon gas leading to Bose-Einstein condensation. Phys. Rev. Lett. 99, 037205 (2007).

8. S.M. Rezende. Theory of coherence in Bose-Einstein condensation phenomena in a microwave-driven interacting magnon gas. Phys. Rev. B 79, 174411 (2009).

9. M.-O. Mewes, M.R. Andrews, D.M. Kurn, D.S. Durfee, C.G. Townsend, W. Ketterle. Output coupler for BoseEinstein condensed atoms. Phys. Rev. Lett. 78, 582 (1997).
10. Immanuel Bloch, Theodor W. Hänsch, Tilman Esslinger. Atom laser with a cw output coupler. Phys. Rev. Lett. 82, 3008 (1999).

11. S.O. Demokritov, V.E. Demidov. Micro-brillouin light scattering spectroscopy of magnetic nanostructures. IEEE Trans. Mag. 44, 6 (2008).

12. S.M. Rezende, F.R. Morgenthaler. Frequency conversion of spin waves in pulsed magnetic fields. Appl. Phys. Lett. 10, 184 (1967).

13. S. Jochim, M. Bartenstein, A. Altmeyer, G. Hendl, S. Riedl, C. Chin, J. Hecker Denschlag, R. Grimm. BoseEinstein condensation of molecules. Science 302, 2101 (2003).

14. J. Klaers, J. Schmitt, F. Vewinger, M. Weitz. Bose-Einstein condensation of photons in an optical microcavity. Nature 468, 545 (2010).

15. S. Christopoulos, G. Baldassarri Höger von Högersthal, A.J.D. Grundy, P.G. Lagoudakis, A.V. Kavokin, J.J. Baumberg, G. Christmann, R. Butté, E. Feltin, J.-F. Carlin, N. Grandjean. Room-temperature polariton lasing in semiconductor microcavities. Phys. Rev. Lett. 98, 126405 (2007).

16. P. Bhattacharya, B. Iao, Ayan Das, S. Bhowmick, J. Heo. Solid state electrically injected exciton-polariton laser. Phys. Rev. Lett. 110, 206403 (2013).

17. J. Kasprzak, M. Richard, S. Kundermann, A. Baas, P. Jeambrun, J.M.J. Keeling, F.M. Marchetti, M.H. Szyman'ska, R. Andre', J.L. Staehli, V. Savona, P.B. Littlewood, B. Deveaud and Le Si Dang. Bose-Einstein condensation of exciton polaritons. Nature 443, 409 (2006).

18. S.O. Demokritov, V.E. Demidov, O. Dzyapko, G.A. Melkov, A.N. Slavin. Quantum coherence due to Bose-Einstein condensation of parametrically driven magnons. New J. Phys. 10, 045029 (2008).

19. P. Nowik-Boltyk, O. Dzyapko, V.E. Demidov, N.G. Berloff, S.O. Demokritov. Spatially non-uniform ground state and quantized vortices in a two-component Bose-Einstein condensate of magnons. Nature Sci. Rep. 2, 482 (2012).

Received 12.09.19

П. Новік-Болтик, І.В. Борисенко,

В.Е. Демидов, С.О. Демокритов

\section{МАГНОННИЙ ЛАЗЕР}

Р е з ю м е

Експериментально продемонстровано можливість створення магнонного лазера на базі когерентного бозеейнштейнівського конденсату магнонів, рух яких був зумовлений використанням змінного в часі поперечно неоднорідного магнітного поля. Показано, що застосування імпульсів поля призводить до формування хмари конденсату, що рухається зі сталою швидкістю $930 \mathrm{~m} / \mathrm{c}$ за використаних в експерименті параметрів. Кількість магнонів, що утворюють хмару, не змінюється з їі поширенням, що нагадує ефект надплинності магнонів. 